\title{
A Graph-Based Approach for Shape Skeleton Analysis
}

\author{
André R. Backes ${ }^{1}$ and Odemir M. Bruno ${ }^{2}$ \\ 1 Instituto de Ciências Matemáticas e de Computação (ICMC) \\ Universidade de São Paulo (USP) \\ Avenida do Trabalhador São-carlense, 400 \\ 13560-970 São Carlos SP Brazil \\ backes@icmc.usp.br \\ 2 Instituto de Física de São Carlos (IFSC) \\ Universidade de São Paulo (USP) \\ Avenida do Trabalhador São-carlense, 400 \\ 13560-970 São Carlos SP Brazil \\ bruno@ifsc.usp.br
}

\begin{abstract}
This paper presents a novel methodology to shape characterization, where a shape skeleton is modeled as a dynamic graph, and degree measurements are computed to compose a set of shape descriptors. The proposed approach is evaluated in a classification experiment which considers a generic set of shapes. A comparison with traditional shape analysis methods, such as Fourier descriptors, Curvature, Zernike moments and Multi-scale Fractal Dimension, is also performed. Results show that the method is efficient for shape characterization tasks, in spite of the reduced amount of information present in the shape skeleton.
\end{abstract}

Keywords: graph, Shape Analysis, Skeleton.

\section{Introduction}

Skeletonization is a method that transforms the shape into a simple structure, which is capable of represent it. The goal of the method is to reduce the information of the shape, in order to make easier the process of recognition and analysis. There are distinct methods for skeleton and most of them are based on the Medial Axis Transform (MAT) originally proposed by Blum 12. The MAT of a shape consists of a set of center coordinates of circles that (i) have to be bi-tangents to the shape and (ii) be completely inside of the shape.

The structure formed by the MAT is a set of interconnected points that represents the original shape with a reduced amount of information. Although the skeleton of a shape is simpler than its contour, the nature of its structure is not so easy to process, and it demands a special analysis. There are some traditional approaches to analyze and recognize skeletons: (i) metrics, this is the simplest 
approach, and it consists of measuring the skeleton structure (i.e., angles formed by lines, width and number of lines); (ii) signal processing based methods, where the contour of the skeleton structure is analyzed by signal processing techniques, such as Fourier transform or wavelets; (iii) fractals [3] and shape methods [4] and (iv) neural networks or other AI techniques, which consists in applying the skeleton directly into the input of a neural network [56].

In this work, we propose a graph-based approach to analyze the skeleton. The method was originally proposed to deal with shape contours [7] and in this work, we propose its use in the skeleton analysis. Experiments compare the method with contour based methods [8], signal processing [9] and fractal dimension [10]. Results demonstrate that the method is suitable to the skeleton analysis and overcome traditional contour approaches.

\section{Shape Skeleton Graph}

\subsection{Shape Skeleton as a Graph}

In order to represent the shape skeleton computed from a shape image as a graph (Figure 1), we consider skeleton information as a list of connected points $S, S=\left[s_{1}, s_{2}, \ldots, s_{N}\right]$, where $N$ is the number of points in the skeleton and $s_{i}=\left(x_{i}, y_{i}\right)$ represents the discreet coordinate of the point $i$ in the skeleton. A graph $G=(V, E)$ is built by considering each point $s_{i} \in S$ as a vertex in graph $G$, i.e., $V=S$. For each pair of vertices $\left(v_{i}, v_{j}\right)$ a non-directed edge $e_{i j} \in E$ is built connecting these vertices. The weight of the edges is computed considering the Euclidean distance between each pair of points of the skeleton:

$$
d\left(s_{i}, s_{j}\right)=\sqrt{\left(x_{i}-x_{j}\right)^{2}+\left(y_{i}-y_{j}\right)^{2}} .
$$

A $N \times N$ matrix $W$ represents the weight of the edges between vertices

$$
W(i, j)=w_{i j}=d\left(s_{i}, s_{j}\right),
$$

where the weights are normalized into the interval $[0,1]$,

$$
W=\frac{W}{\max _{w_{i j} \in W}} .
$$

The resulting graph presents a regular behavior as each vertex presents the same characteristic, such as number of connections, as other vertices. Hence, no relevant properties can be computed from this graph. Thus, a transformation must be applied over this graph to achieve relevant properties that can be used to characterize this graph structure and, as a result, the original shape skeleton. For this transformation, we propose to use the dynamic evolution of the graph. 


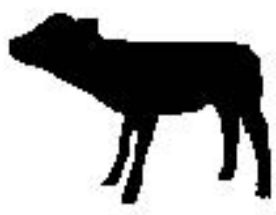

(a)

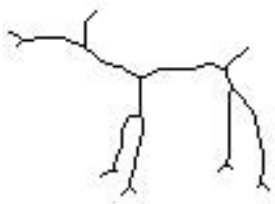

(b)

Fig. 1. (a) Original shape; (b) Shape skeleton

\subsection{Dynamic Evolution}

Graphs with different topological aspect present a large range in their characteristics. Besides, the interaction between structural and dynamical aspects improve graph characterization [1].

Modeling the dynamics of a graph is a difficult task. It may involve node addition or deletion, changes in edges positions or anything else, during a time period. However, the graph structure dynamics can be estimated by applying a transformation over the original graph. Then, properties from this new version of the graph are computed and used to characterize its dynamics [12.

A graph transformation can be performed in many ways. A very simple and straight approach is to apply a threshold $t$ over the set of edges $E$ to select $E^{*}$, $E^{*} \subseteq E$. By keeping the original set of nodes $V$, a new graph $G^{*}=\left(V, E^{*}\right)$, which represents an intermediary stage in graph evolution, is achieved.

In our approach, an edge belongs to $E^{*}$ with its weight is equal or smaller than a threshold $t$ (Figure 2). This $\delta_{t}$ transformation can be represented as

$$
E^{*}=\delta_{t}(E)=\{e \in E \mid w(e) \leq t\} .
$$

By applying different thresholds $t$ over the original graph it is possible to provide a richer set of measurements, thus providing a better graph characterization.

\subsection{Measurements}

Graph theory provides numerous measurements that can be computed from a graph or network. The vertex degree is largely used in graph studies due to its

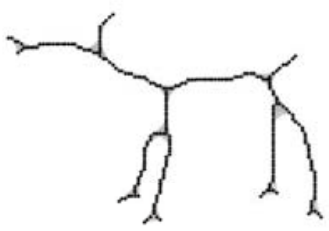

(a)

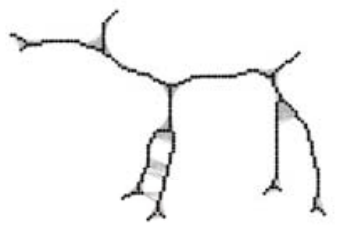

(b)

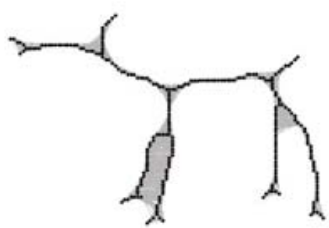

(c)

Fig. 2. Different stages in graph evolution. Edges in each graph are the only ones with weight smaller than a threshold $t$. 
simplicity and for being a characteristic present in any graph. It is defined as the number of incident edges (or connections) in a vertex $v_{i} \in V$. The degree of a vertex $v_{i}, \operatorname{deg}\left(v_{i}\right)$, indicates its connectivity and can be defined as:

$$
\operatorname{deg}\left(v_{i}\right)=\left|\left\{e \in E \mid v_{i} \in e\right\}\right|=\left|\left\{v_{j} \in V \mid\left\{v_{i}, v_{j}\right\} \in E\right\}\right|=\left|\partial v_{i}\right|,
$$

with

$$
\partial v_{i}=\left\{v_{j} \in V \mid\left(v_{i}, v_{j}\right) \in E\right\}
$$

where $\partial v_{i}$ is the set of neighbors of $v_{i}$ and $|A|$ denotes the cardinality (number of elements) of a set $A$ [13.

To characterize a graph, we propose to use global measurements computed from degree distribution analysis. Two measurements have been considered for this application: the Average Degree

$$
A v(G)=\sum_{v_{i} \in V} \frac{\operatorname{deg}\left(v_{i}\right)}{|N|},
$$

and the Maximum Degree

$$
\operatorname{Max}(G)=\max _{i} \operatorname{deg}\left(v_{i}\right)
$$

\section{Skeleton Graph Signature}

We propose to perform skeleton characterization by using a signature which describes temporary characteristics of the computed skeleton graph. This signature is a feature vector $\varphi$ composed by Average and Maximum Degree measurements computed from each transformed graph $G^{*}$ achieved by applying a transformation $\delta_{t}$ over the original graph $G$, for different values of $t \in T$ :

$$
\varphi=\left[\operatorname{Av}\left(G_{t_{1}}\right), \operatorname{Max}\left(G_{t_{1}}\right), \ldots, A v\left(G_{t_{M}}\right), \operatorname{Max}\left(G_{t_{M}}\right)\right], t_{i} \in T,
$$

where $G_{t_{i}}$ is the graph $G^{*}$ computed using a threshold $t_{i}$.

In order to avoid scale distortions, the proposed signature is normalized by the number of vertices present in the graph, $N$. So, degree measurements are represented as proportions of the original graph.

\section{Experiments}

To evaluate the performance of the proposed method, experiments have been done using a set of artificial shape images. This shape database consists of 9 different classes with 11 samples each, which makes a total of 99 images [14 15. Each class groups different variations of a same shape structure, such as occlusion, articulation and missing parts (Figure 33).

Skeleton graph signature was computed considering different manifestations of the skeleton graph during its dynamic evolution. Thus, to compose the signature, 


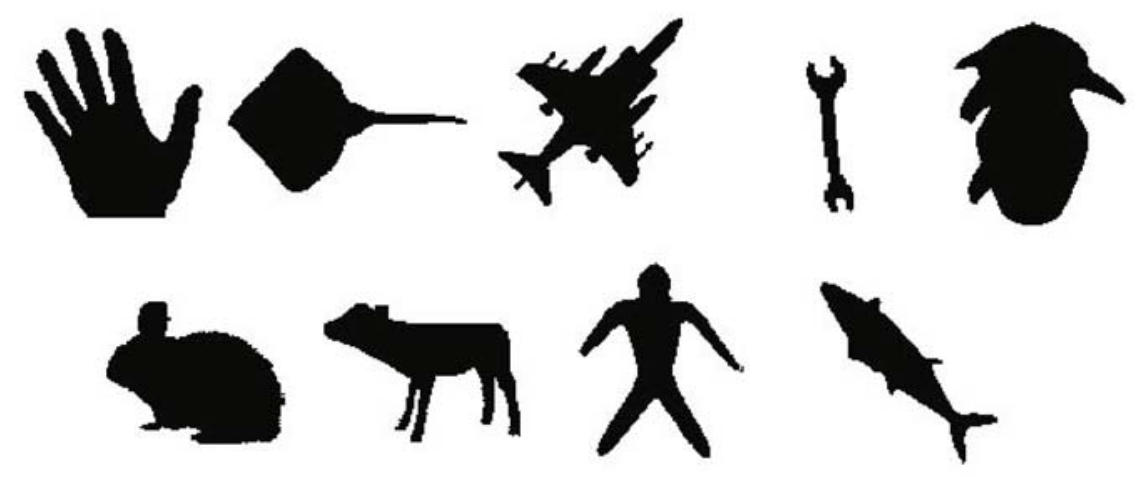

Fig. 3. Example of the artificial shapes used in the experiments

the thresholds $t \in\{0.1,0.21,0.32,0.43,0.54,0.65\}$ were used. For each new graph achieved, the maximum and average degrees were computed to compose the skeleton signature, totalizing 12 descriptors.

The proposed signature was evaluated using Linear Discriminant Analysis (LDA) in a leave-one-out cross-validation scheme [1617]. This method has as objective to find a linear sub-space to project the data where variance intraclasses is larger than inter-classes.

To provide a better evaluation, proposed signature was compared with traditional shape analysis methods from literature. So, the following methods were considered in this experiment:

Fourier descriptors: this approach uses a feature vector containing the 20 most significant coefficients of the Fourier Transform computed from the shape contour, as described in 918.

Zernike moments: a total of 20 moments (order $n=0, \ldots, 7$ ) are used to compose a feature vector. Each represents the most significant magnitudes computed from a set of orthogonal complex moments of the shape image 19.

Curvature descriptors: this approach uses a curve to represent the contour of a shape. Maximum and minimum local points of this curve correspond to the direction changes in the shape contour [8].

Multi-scale Fractal Dimension: a curve is used to describe how the shape complexity changes along the scale. In this approach shape characterization is performed using the 50 most meaningful points of the curve 310].

Skeleton Paths: from a shape skeleton, this method uses the shortest path between each pair of end Points of the skeleton to describe it. Each shortest path is described by $M$ equidistant skeleton points. Each point is represented by the maximum disc size associated to it. Unlike the other methods, LDA was not used for classification, but a graph matching approach which estimates the probability of one path belonging to a specific shape skeleton [20]. A total of 15 equidistant points was used to represent each path. The total number of descriptors of a shape skeleton varies according to the (number of shortest paths in the skeleton) $\times 15$. 


\section{Results}

Table 1 shows the results yielded for each method in the proposed experiment. The proposed approach uses shape skeleton for characterization and identification of shape patterns. Its comparison was carried out considering contourbased approaches. Shape contours are able to represent more efficiently all details present in a shape than its skeleton. Besides, shape skeletons are more sensitive to variations or noise in the surface of the shape, i.e., small changes in the shape may produce abrupt changes in the skeleton. In our approach, we computed the shape skeleton using method by Choi et al. 21]. A pruning method 22 is applied over the resulting skeleton to minimize shape deformation and noise. Thus, only the most significant ending branches remain in the skeleton.

Results show that, in spite of the reduced amount of information present in the shape skeleton and its deficiencies in shape representation, the proposed method presents great capacity for shape recognition, overcoming traditional contour-based methods, such as Fourier descriptors, Zernike moments, Curvature descriptors and Multi-scale Fractal Dimension. Using simple degree-based measurements computed from the skeleton graph, the proposed approach can characterize different shape patterns with different variations in their structure (such as, occlusion, articulation and missing parts), confirming the great efficacy of the method.

The proposed approach also present a superior performance in comparison to another skeleton-based method, the skeleton paths. Our approach uses all skeleton points to compute a graph whose properties are used to describe it and, as a consequence, the original shape. The skeleton path method uses the shortest paths (sampled by $M$ points) computed between two end Points to describe the skeleton. However, we have that skeletons from different shapes may present similar groups of paths (Figure 4). Thus, the information of how skeleton points are distributed over the skeleton is quite important to the effectiveness of the method. It is a characteristic present in our approach which explains the superiority of our approach to the skeleton paths method.

Table 1. Classification performance of various shape descriptors for the artificial shapes database

\begin{tabular}{|c|c|c|}
\hline $\begin{array}{c}\text { Shape } \\
\text { Descriptor }\end{array}$ & $\begin{array}{c}\text { Shapes correctly } \\
\text { classified }\end{array}$ & $\begin{array}{c}\text { Mean success } \\
\text { rate }(\%)\end{array}$ \\
\hline Proposed Method & 93 & 93.94 \\
\hline Fourier descriptors & 83 & 83.84 \\
\hline Zernike moments & 91 & 91.92 \\
\hline Curvature & 76 & 76.77 \\
\hline M. S. Fractal Dimension & 87 & 87.88 \\
\hline Skeleton Path & 91 & 91.92 \\
\hline
\end{tabular}




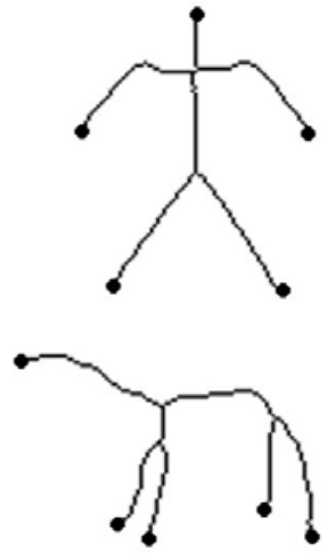

(a)
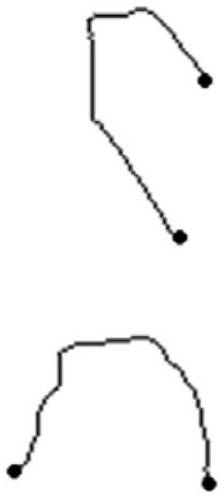

(b)
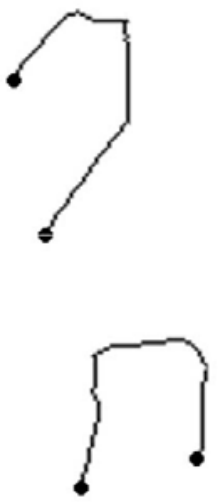

(c)

Fig. 4. (a) Skeletons of two different shapes; (b)-(c) Similar paths in the skeletons

\section{Conclusion}

Paper presents a novel approach for pattern characterization using shape skeletons. There, shape skeleton is represented and characterized as a graph in a dynamic evolution context. Degree based measurements (such as average and maximum degrees) are used to characterize this graph and, as a consequence, the original shape. Results reported a powerful potential of discriminating shape patterns, including those with different variations in their structure, overcoming traditional shape analysis (such as curvature, Fourier descriptors, Zernike moments and Multi-scale Fractal Dimension) and skeleton-based methods (Skeleton paths).

\section{Acknowledgements}

A.R.B. acknowledges support from FAPESP (2006/54367-9). O.M.B. acknowledges support from CNPq (306628/2007-4).

\section{References}

1. Blum, H.: A transformation for extracting new descriptors of shape. In: WathenDunn, W. (ed.) Models for the Perception of Speech and Visual Forms, pp. 362-380. MIT Press, Amsterdam (1967)

2. Blum, H., Nagel, R.: Shape description using weighted symmetric axis features. Pattern Recognition 10(3), 167-180 (1978)

3. da S. Torres, R., Falcão, A.X., da F. Costa, L.: A graph-based approach for multiscale shape analysis. Pattern Recognition 37, 1163-1174 (2003) 
4. Sebastian, T.B., Kimia, B.B.: Curves vs. skeletons in object recognition. Signal Processing 85(2), 247-263 (2005)

5. Wang, C., Cannon, D.J., Kumara, S.R.T., Guowen, L.: A skeleton and neural network-based approach for identifying cosmetic surface flaws. IEEE transactions on neural networks 6(5), 1201-1211 (1995)

6. Zhu, X.: Shape recognition based on skeleton and support vector machines. In: Third International Conference on Intelligent Computing, pp. 1035-1043 (2007)

7. Backes, A.R., Casanova, D., Bruno, O.M.: A complex network-based approach for boundary shape analysis. Pattern Recognition 42(1), 54-67 (2009)

8. Wu, W.Y., Wang, M.J.: On Detecting the dominant points by the curvature-based polygonal approximation. CVGIP: Graphical Models Image Process 55, 79-88 (1993)

9. Gonzalez, R.C., Woods, R.E.: Digital Image Processing, 2nd edn. Prentic-Hall, New Jersey (2002)

10. Plotze, R.O., Padua, J.G., Falvo, M., Vieira, M.L.C., Oliveira, G.C.X., Bruno, O.M.: Leaf shape analysis by the multiscale minkowski fractal dimension, a new morphometric method: a study in passiflora 1 (passifloraceae) 83, 287-301 (2005)

11. Boccaletti, S., Latora, V., Moreno, Y., Chavez, M., Hwang, D.U.: Complex networks: Structure and dynamics. Physics Reports 424(4-5), 175-308 (2006)

12. da F Costa, L., Rodrigues, F.A., Travieso, G., Villas Boas, P.R.: Characterization of complex networks: A survey of measurements. Advances in Physics 56(1), 167-242 (2007)

13. Wuchty, S., Stadler, P.F.: Centers of complex networks. Journal of Theoretical Biology 223, 45-53 (2003)

14. Sebastian, T.B., Klein, P.N., Kimia, B.B.: Recognition of shapes by editing their shock graphs. IEEE Trans. Pattern Analysis and Machine Intelligence 26(5), 550-571 (2004)

15. Sharvit, D., Chan, J., Tek, H., Kimia, B.B.: Symmetry-based indexing of image databases. Journal of Visual Communication and Image Representation 9(4), 366-380 (1998)

16. Everitt, B.S., Dunn, G.: Applied Multivariate Analysis, 2nd edn. Arnold (2001)

17. Fukunaga, K.: Introduction to Statistical Pattern Recognition, 2nd edn. Academic Press, London (1990)

18. Osowski, S., Nghia, D.D.: Fourier and wavelet descriptors for shape recognition using neural networks - a comparative study. Pattern Recognition 35(9), 1949-1957 (2002)

19. Zhenjiang, M.: Zernike moment-based image shape analysis and its application". Pattern Recognition Letters 21(2), 169-177 (2000)

20. Bai, X., Latecki, L.J.: Path similarity skeleton graph matching. IEEE Transactions on Pattern Analysis and Machine Intelligence 30(7), 1282-1292 (2008)

21. Choi, W.P., Lam, K.M., Siu, W.C.: Extraction of the euclidean skeleton based on a connectivity criterion. Pattern Recognition 36(3), 721-729 (2003)

22. Bai, X., Latecki, L.J., Liu, W.Y.: Skeleton pruning by contour partitioning with discrete curve evolution. IEEE Trans. Pattern Analysis and Machine Intelligence 29(3), 449-462 (2007) 